\title{
INFLUÊNCIA DO RISCO FINANCEIRO NA CRIAÇÃO DE VALOR NA INDÚSTRIA ALI- MENTÍCIA DE EMPRESAS LISTADAS NA B3 ${ }^{1}$
}

\section{INFLUENCE OF FINANCIAL RISK ON VALUE CREATION IN THE FOOD INDUSTRY OF COMPANIES LISTED ON B3}

\author{
David Ferreira Lopes Santos \\ Doutor em Administração (Mackenzie) \\ Universidade Estadual Paulista (UNESP) \\ david.lopes@fcav.unesp.br \\ Gabriela Oliveira de Sales \\ Graduada em Administração (UNESP) \\ Universidade Estadual Paulista \\ gabi.oliveirasaless@gmail.com \\ Henrique de Santana Rangel \\ Mestrando em Administração (UNESP) \\ Universidade Estadual Paulista \\ henrique.rangel@hotmail.com \\ Stela Basso Montoro \\ Doutoranda em Agronomia (UNESP) \\ Universidade Estadual Paulista \\ stelamontoro@hotmail.com
}

\begin{abstract}
RESUMO
Objetivo: Identificar a influência do risco financeiro na criação de valor na indústria alimentícia de empresas listadas na B3.

Fundamento: $\mathrm{O}$ risco é um fator inerente às decisões de investimentos e endividamento das empresas, sendo que os métodos de mensuração e os modelos empíricos são temas em discussão na literatura. Entende-se que estratégias empresariais podem influenciar na redução de assimetrias e na menor exposição dos ativos ao risco sistemático, o que resultaria na influência positiva na criação de valor. No entanto, esses pressupostos carecem de evidências empíricas, especialmente, quando voltadas para segmentos específicos e que exponham as características individuais das empresas.
\end{abstract}

\footnotetext{
${ }_{1}^{1}$ Artigo recebido em: 27/01/2020. Revisado por pares em: 24/09/2020. Reformulado em: 26/09/2020. Recomendado para publicação: 30/09/2020 por Marco Aurélio dos Santos (Editor Adjunto). Publicado em: 11/02/2021. Organização responsável pelo periódico: UFPB
} 
Método: A partir da abordagem da relação entre risco e criação de valor, utilizou-se uma análise de regressão para verificar o impacto do risco nas métricas de criação de valor no setor alimentício. O estudo foi realizado a partir de informações econômicas e financeiras, com dados disponíveis entre 2013 e 2018. Os resultados foram construídos por meio da análise de regressão com dados em painel, utilizando a técnica de mínimos quadrados ponderados.

Resultados: A análise realizada apontou que os riscos presentes no ambiente pertinentes às empresas estabelecidas no Setor Alimentício e listadas na Brasil Bolsa Balcão (B3) impactam negativamente na criação de valor. Os resultados operacionais e financeiros das empresas, estão diretamente relacionados à criação de valor por meio de indicadores como o $\mathrm{EVA}^{\circledR} \mathrm{e}^{\mathrm{o}} \mathrm{MVA}^{\circledR}$. Tais indicadores mostram que a importâncias dos resultados operacionais e estratégias gerenciais que reduzam assimetrias para diminuir a volatilidade dos ativos que impacta negativamente no valor das empresas.

Contribuições: Inúmeras pesquisas exploram abordagens de gestão de risco e retorno dos negócios. Este artigo permite evidenciar que a mitigação do risco pode maximizar a criação de valor. Para tal, a implementação de melhorias dos aspectos relacionados à governança, ética e transparência do negócio como estratégia podem reduzir as assimetrias e os impactos do risco da indústria alimentícia. A criação de valor deve ser o objeto principal da gestão financeira de uma empresa e é a partir do conhecimento do valor de um negócio que é possível tomar diversas decisões.

Palavras-chave: Criação de Valor. Risco Financeiro. Indústria Alimentícia.

\section{ABSTRACT}

Objective: To identify the influence of financial risk on value creation in the food industry of companies listed on B3.

Foundations: Risk is an inherent factor in companies' investment and debt decisions, with measurement methods and empirical models being discussed in the literature. It is known that business strategies can influence the reduction of asymmetries and the lower exposure of assets to systematic risk, which would result in a positive influence on the value creation. However, these assumptions lack empirical evidence, especially when focused on specific segments and that expose the individual characteristics of the companies.

Method: From the approach of the relationship between risk and value creation, a regression analysis was used to verify the impact of risk on the value creation metrics in the food sector. The study was conducted based on economic and financial information, with data available between 2013 and 2018. The results were constructed using regression analysis with panel data, using the weighted least squares technique.

Results: The analysis carried out showed that the risks present in the environment pertaining to companies established in the Food Sector and listed on Brasil Bolsa Balcão (B3) have a negative impact on the creation of value. In this context, the companies' operating and financial results are directly related to the creation of value through indicators such as EVA ${ }^{\circledR}$ and MVA ${ }^{\circledR}$. These indicators show the importance of operating results and management strategies that reduce asymmetries to decrease assets volatility which negatively impacts the value of companies.

Contributions: Several surveys explore approaches to risk management and business return. This article shows that risk mitigation can maximize the creation of value. To this end, the implementation of improvements in aspects related to business governance, ethics and transparency as a strategy can reduce the asymmetries and the impact of risk in the food industry. The creation of value must be the main object of the financial management of a company and it is from the knowledge of the value of a business that it is possible to make several decisions.

Keywords: Value Creation. Financial risk. Food industry. 


\section{INTRODUÇÃO}

Em mercados competitivos a obtenção de capital para o financiamento das atividades empresariais requer uma estratégia de investimentos que direcione os recursos obtidos para ativos que possam oferecer um retorno superior ao risco assumido pelos credores e acionistas (Corrêa, Assaf Neto \& Lima, 2013).

Dessa forma, quanto maior for as variações e incertezas dos fluxos de caixa futuros de um investimento ou negócio, maior serão os riscos. Por isso, investidores se preocupam em mensurar os riscos inerentes as suas aplicações e exigem uma remuneração diretamente proporcional ao nível de risco associado ao investimento realizado (Teixeira, 2014).

O risco de uma empresa pode ser mensurado de diferentes formas, sendo que as fontes de variabilidade de resultados futuros podem ser exógenas e endógenas às empresas (Silveira, Santos, \& Rodrigues, 2017). Os fatores externos às empresas que podem influir nas suas decisões empresariais e no seu risco são do âmbito econômico, político, sociodemográfico, internacional ou tecnológico (Kapustina et al., 2018).

No que diz respeito aos fatores endógenos, encontram-se: maturidade e expertise do seu processo gerencial e gestores; estrutura de governança; competência e habilidades dos funcionários e da cultura organizacional; domínio tecnológico dos ativos tangíveis; domínio gerencial e operacional dos ativos intangíveis; e a estrutura financeira quanto aos seus níveis de alavancagem, folga e rentabilidade (Aguiar, Pimentel \& Resende, 2011).

A criação de valor é objeto principal da gestão financeira de uma empresa. Somente conhecendo a capacidade de geração de rentabilidade e valor de um negócio, que é possível tomar decisões as quais incluem: atrair investidores para comercialização das ações da empresa, atrair assessoria de banco de investimento para captação de recursos, escolher se é melhor aumentar o investimento na empresa ou investir em uma nova, entre outras decisões pertinentes a continuidade do negócio (Lunardi et al. 2017)

Deste modo, o maior risco percepcionado pelos acionistas determinará o retorno exigido relativos a empresa, podendo ocasionar a esta: a) necessidades de gerar maiores fluxos financeiros; b) dificuldades na obtenção de retornos; c) dificuldades de encontrar novos financiadores e investimentos (Teixeira, 2014). Tais fatores evidenciam a importância de ampliar a compreensão do risco nas estratégias de investimentos e negócios no que concerne à mensuração do seu impacto econômico (Maria, 2018). Na medida em que os valores dos ativos precisem ser apurados pelo seu valor econômico, o melhor entendimento dos reflexos do risco na criação de valor poderá auxiliar na avaliação dos modelos de valoração dos ativos das empresas.

Os fundamentos econômicos e financeiros inerentes às empresas são premissas importantes analisadas pelo mercado, ao mesmo tempo em que a medida de criação de valor é responsável por controlar e indicar a consistência e desempenho das empresas, considerando a necessidade de criar valor há uma demanda cada vez maior por uma gestão voltada para o valor de mercado (Silva \& Santos, 2015; Santos, Tavares, Azevedo \& Freitas Neto, 2018).

As empresas brasileiras têm buscado diferentes estratégias para o financiamento dos seus investimentos, a fim de diversificar seus riscos (Costa, Almeida Ferreira \& Silva, 2013). Atribui-se a ascensão do gerenciamento de risco: a) mudança no ambiente competitivo (Vilhena \& Camargos 2014); b) sofisticação tecnológica (Bali \& Zhou 2016); c) interconectividade global (Degenhart et al., 2017). Em função disso, o ambiente organizacional tornou-se mais complexo e a contabilidade, a gestão de riscos e a governança corporativa, cada vez mais, interligadas e interdependentes (Bhimani, 2009).

A indústria alimentícia é objeto deste estudo devido a sua abrangência e importância para a economia de qualquer país, sendo formada por uma rede de setores relacionados, como o agrícola, o de serviços e insumos, aditiva, fertilizantes, agrotóxicos e bens de capital (Gouveia, 2006). Além 
disso, o setor tem fundamental importância social e econômica no Brasil representando um papel importante para o cenário econômico (Raimundo, Batalha, \& Torkomian, 2017).

Atualmente, o setor é o maior em valor bruto de produção da indústria de transformação (Raimundo et al., 2017). Diante disso, há uma relevante necessidade em se avaliar o desempenho das empresas alimentícias, a qual se encontra associada ao agronegócio brasileiro, um setor transversal com atuação em diferentes cadeias produtivas (Silveira et al., 2017).

Não obstante, há poucos estudos na área de finanças focados no segmento alimentício, o que aumenta a originalidade deste estudo ao abordar o desempenho ajustado ao risco e sua influência na criação de valor das empresas deste setor (Raimundo et al., 2017). Assim, o objetivo desse trabalho é identificar a influência do risco financeiro na criação de valor na indústria alimentícia, representada pelas empresas com ações negociadas na Bolsa de Valores de São Paulo (B3), no período de 2013 a 2018.

\section{FUNDAMENTO EMPÍRICO DA PESQUISA}

\subsection{Risco Financeiro}

Aplicando o conceito de risco à realidade das empresas, há alguns tipos de risco que em conjunto representam o risco total que irá incorrer no ativo da empresa:

- O risco específico, que está associado à atividade específica da empresa e à sua posição competitiva em relação aos seus concorrentes diretos;

- O risco do mercado, que está diretamente relacionado ao setor em que a empresa está inserida, e ao modo como os contextos econômico, político e legal, social e tecnológico, poderão influenciar o seu sucesso empresarial (Bali \& Cakici, 2009).

Em relação ao risco específico da empresa, pode-se dividi-lo em:

- O risco do negócio, que está associado à atividade de exploração da empresa, avaliando de que forma a estratégia e as ações implementadas permitem que o negócio tenha capacidade de autofinanciamento, ou seja, cubra a totalidade de gastos necessários para realização normal das atividades da empresa;

- O risco financeiro, que está interligado com a estrutura financeira da empresa, devendo ser analisado em três perspectivas, como a cobertura dos encargos financeiros, a tesouraria e a estrutura de capitais (Bartram, Brown, \& Waller, 2015).

Assim, o trade-off risco versus retorno representa um papel determinante na escolha do destino dado ao capital privado, sendo que os investimentos se tornam mais atrativos a medida que essas duas variáveis assumem uma posição coerente (Bali \& Zhou, 2016).

De maneira geral, o risco pode ser considerado uma medida de incerteza, assim, em um dado acontecimento ou comportamento é possível realizar uma previsão sobre o fato e ter mais ou menos incerteza, sendo necessário considerar um determinado intervalo de tempo, visto que se as previsões forem feitas para um futuro muito distante, novas variáveis são acrescentadas, tornando mais arriscados os valores previstos (Silva, Nardi, \& Pimenta Junior, 2012).

Entretanto, o risco não deve ser entendido apenas como algo negativo, isso porque a possibilidade de ganho além do previsto também o compõe (Bali \& Zhou, 2016). Desse modo, diante de uma variedade de modelos de avaliação de performance, é importante a definição de qual método é o mais apropriado (Gaspar, Santos, \& Rodrigues, 2014).

O Capital Asset Pricing Model (CAPM) estabelece que a taxa de retorno esperado dos ativos com riscos está relacionada linearmente a taxa livre de ricos e a taxa de retorno esperada, verificando se o título está sendo negociado com um valor justo. A diferença entre o retorno esperado e aquele livre de risco é denominado prêmio pelo risco, funcionando com um retorno adicional que mede a remuneração do risco que um investidor suporta para concordar com determinado grau de risco quando investe seu capital (Fama \& French, 2004) 
O Information Ratio (IR) relaciona o retorno do ativo com o risco. Em uma medida inversa ao coeficiente de variação, o IR mensura o retorno obtido para cada unidade de risco assumido, permitindo de uma maneira direta comparar o desempenho de diferentes ativos.

O Índice de Treynor (IT) é um indicador de avaliação do desempenho de fundos de investimentos que faz uso do risco sistemático do ativo ou fundos para sinalizar a ligação entre o risco sistemático ( $\beta$ ) e o retorno excedente do ativo (Treynor, 1962). O coeficiente $\beta$ corresponde ao risco sistemático definido pelo CAPM (Silva, Nogueira \& Ribeiro, 2015).

O Índice de Sharpe (IS), por intermédio de uma equação relaciona o risco e o retorno de um ativo específico e indica se a rentabilidade esperada é compatível com o risco exposto, nesse sentido, quanto maior o valor do índice mais atrativo se torna o ativo (Silva et al., 2015). De acordo com Oliveira Filho e Sousa $(2015$, p. 65) este indicador "mede o retorno excedente do portfólio em relação à taxa livre de risco, também chamado de prêmio de risco, medido por seu desvio-padrão, que representa o risco total do portfólio". Segundo Gaspar et al., (2014) este índice assemelha-se ao modelo de Treynor, a única diferença é o denominador.

Luz (2013) descrevem que o risco de mercado, mensurado pela volatilidade (desviopadrão) dos preços de ativos ou das taxas de retorno, é um fator que pode influenciar a rentabilidade e a liquidez, e a relação entre as duas. Recentes crises financeiras têm motivado a realização de pesquisas sobre esta questão nos últimos anos, as quais apontam que o risco de mercado reduz a liquidez nas empresas e este fenômeno pode ser explicado pela limitação de recursos e restrições de crédito no mercado financeiro.

O efeito conjunto da rentabilidade e do risco de mercado sobre a liquidez é positivo na maioria de subgrupos de empresas, estes resultados são também consistentes com o fato de que as empresas com rentabilidades maiores têm melhores condições de aumentar a liquidez por precaução do que as empresas com menores rentabilidades nos momentos de crise.

\subsection{Criação de Valor}

O valor de mercado de uma determinada empresa de capital aberto representa a expectativa dos investidores sobre a sua geração futura de caixa, assumindo que o mercado seja eficiente, o preço da ação deve refletir todas as informações que afetem esse fluxo futuro de caixa. Dessa forma, o valor de mercado de uma empresa deve refletir, em equilíbrio, o fluxo de caixa descontado de uma firma. Como resultado, a relação entre o valor de mercado sobre o valor contábil de uma empresa representa o seu nível de oportunidades de crescimento, quanto maior esse indicador, maiores seriam as oportunidades de crescimento (Kayo, 2015).

O valor dos ativos é determinado por alguns fatores, entre os quais se encontra a estratégia competitiva, que é "formulada e implementada com a finalidade de gerar valor para o acionista, pois diante de um ambiente competitivo e caracterizado pela incerteza, os gestores buscam estratégicas que maximizem o retorno para o acionista" (Aguiar et al., 2011).

Na Gestão Baseada em Valor, a meta mais importante é a maximização da riqueza dos acionistas, isto é, aumentar o valor econômico agregado dos investidores da empresa (Silva et al., 2018). Ao aplicarem recursos nas empresas os investidores do mercado acionário buscam, principalmente, "o valor presente dos fluxos futuros de benefícios econômicos que a empresa pode produzir" (Martins, 2009, p. 18), ou seja, sua criação de valor. A maximização da riqueza dos acionistas implica na maximização da diferença entre o valor da empresa e o capital investido, tornando fundamental uma gestão empresarial com base na criação de valor (Araújo \& Assaf Neto, 2003).

A visão "Porteriana" admite que o valor total criado nas empresas é a diferença entre o valor criado e o custo das atividades, assim, se o desempenho das atividades for mantido, a minimização dos custos proporciona o aumento do valor total (Toledo \& Mello, 2013). Sob outra perspectiva, Brito e Brito (2012) mostram que o valor criado não se refere necessariamente ao valor apro- 
priado, uma vez que o custo de oportunidade e disposição a pagar definem o valor criado, enquanto preço e custo definem o valor apropriado.

Mensurar a criação de valor é possível por meio dos indicadores de valor, sendo que para a determinação correta e efetiva dessa mensuração, Silva et al., (2018) apresentam três princípios baseados em índices de desempenho financeiro e operacionais: (1) os direcionadores de valor devem estar diretamente ligados à criação de valor para o acionista e serem aplicados em toda organização; (2) os direcionadores de valor devem ser utilizados como importantes medidas financeiras e operacionais; (3) os direcionadores devem englobar o crescimento de longo prazo e o desempenho operacional.

Empresas com gestão estratégica e empresarial apresentam performance assertiva e maior capacidade de criação valor, gerando vantagem competitiva frente à concorrência, ao passo que a superioridade na criação de valor propicia capacidade de manobra, resultados superiores e crescimento de mercado (Brito \& Brito, 2012; Carvalho, Kayo, \& Martin, 2010). Em uma relação entre o desempenho da empresa e a vantagem competitiva, a gestão estratégica é o ponto de partida, a partir do qual se gera um desempenho empresarial diferenciado responsável pela competitividade.

Nas últimas duas décadas a gestão baseada no valor tem ganhado evidência na literatura acadêmica, e a medição do desempenho empresarial interno e externo é inovado com a determinação do EVA ${ }^{\circledR}$ (Economic Value Added) (Santos, 2005; Lunardi et al., 2015; Silva \& Santos 2015; Santos et al., 2018).

No que diz respeito ao estudo de indicadores no mercado financeiro, o índice P/L (preço/lucro) é um dos mais utilizado entre os métodos de avaliação relativa. Sua funcionalidade está relacionada ao fato de capturar o risco e o crescimento de uma ação (Alberto \& Vieira, 2011) e é calculado a partir da fórmula (1).

$$
\frac{P_{0}}{L_{0}}
$$

Em que:

$P_{0}=$ Preço por ação no instante 0 ;

$L_{0}=$ Lucro por ação no instante 0 ;

Estudos apontam que há indícios da existência de uma relação entre o índice preço/lucro e o retorno requerido da ação, sendo que, quanto menor o índice $\mathrm{P} / \mathrm{L}$, maior deverá ser o retorno, do mesmo modo que maiores retornos estão associados a maiores riscos e assim, conclui-se que ações com baixo P/L sejam mais arriscadas (Alberto e Vieira, 2011).

\subsubsection{Valor Econômico Adicionado (EVA ${ }^{\circledR}$ ) e o Valor de Mercado Adicionado (MVA ${ }^{\circledR}$ )}

$\mathrm{O} \mathrm{EVA}^{\circledR}$ é uma medida de desempenho que demonstra a rentabilidade de um investimento frente a outras opções, ou seja, representa o custo de oportunidade do capital próprio. Segundo Corrêa et al. (2013), este desempenho leva em consideração o RROI (Retorno Residual do Investimento).

Este valor demonstra se a empresa está ganhando, em um exercício, mais do que o custo de capital atribuído aos recursos utilizados. Sua vantagem está em indicar quanto realmente foi agregado de valor na empresa, permitindo à gestão da empresa o discernimento exato se há criação (EVA positivo) ou destruição (EVA negativo) de valor aos acionistas (Lunardi et al., 2017). Portanto, o EVA ${ }^{\circledR}$ é capaz de medir a eficiência da empresa de remunerar seus acionistas acima dos seus custos de investimentos, indicando uma medida de destruição ou criação de valor da empresa (Silva \& Santos, 2015). 
A métrica $E V A \circledast$ pode ser determinada pelos benefícios gerados pelas organizações depois de descontados os custos intrínsecos ao seu funcionamento e os custos de capital investidos nas mesmas. O custo de capital é composto pelos custos de financiamentos por capitais alheios e, também, pelos custos de capital próprio, o que gera uma mensuração mais precisa do lucro das empresas (Lunardi et al., 2017).

Outra medida de valor agregado pelo mercado é chamada de Valor de Mercado Adicionado ou $\mathrm{MVA}^{\circledR}$. Essa métrica retrata a riqueza produzida aos detentores de capital definida pela competência operacional da empresa em gerar resultados mais elevados em relação ao seu custo de oportunidade (Santos 2005). Por outra ótica, esse indicador refere-se ao quanto a empresa possui de valor adicionado em relação a quantidade que deveria desembolsar para comprar o total de seus ativos a custo de mercado (Araújo \& Assaf Neto, 2003).

De acordo com Corrêa et al., (2013), o MVA ${ }^{\circledR}$ é considerado essencial para a análise e avaliação da performance de uma empresa e seu potencial de criação de valor, já que os índices tradicionais de análise dos demonstrativos financeiros posicionam-se de forma restrita na avaliação do conceito em questão. Através do resultado auferido em excesso, obtido pela diferença do investimento total e o valor de mercado, apresenta-se o valor estimável do intangível do negócio, determinando o quanto uma companhia foi eficiente, visando proporcionar riqueza aos seus acionistas (Araújo \& Assaf Neto, 2003).

\subsection{Competitividade no setor alimentício}

De acordo com Farina (1999), a competitividade é definida como a capacidade de uma empresa de sobreviver e, principalmente, crescer em um mercado, seja ele novo ou corrente, visto que o ambiente competitivo é formado pela interação entre a estrutura do mercado, padrões de concorrência e características de demanda. No Brasil, a indústria de alimentos tem utilizado a diferenciação como principal estratégia para atrair novos consumidores, na busca de atingir maior grau de competitividade no mercado (Scagliusi, Machado, \& Torres, 2005).

Outra característica relevante para esta análise trata-se da dimensão deste setor, o qual conta com milhares de estabelecimentos entre pequenas, médias e grandes firmas, presentes em todo o país, além da elevada heterogeneidade das firmas e subsetores em que atuam, como: laticínios, óleos e gorduras, derivados de carne, do trigo, de frutas e vegetais, entre outros, que produzem 850 tipos de produtos (Gouveia, 2006).

Assim, observa-se que os aspectos e requisitos de competição nas empresas alimentícias tornam-se cada vez mais evidentes e, além da diversificação dos produtos e da produção, o mercado de alimentos passa a exigir qualidade em seu portfólio (Biasi et al., 2018). Ainda segundo os autores, as inovações nestas empresas são, em sua grande maioria, incrementais e os investimentos em pesquisa e desenvolvimento (P\&D) são relativamente baixos se comparados com outros setores da indústria brasileira.

Em linhas gerais, trata-se de um mercado altamente competitivo e dinâmico, onde decisores e empresários devem agir rapidamente para acompanhar as mudanças no comportamento do consumidor, portanto, é imperativo inovar, buscando o posicionamento dinâmico dos produtos no mercado, inovando tecnologicamente para manter a organização competitiva em relação à qualidade/custo de seus produtos (Krücken-Pereira, Abreu, \& Bolzan, 2002).

As características apresentadas proporcionam ao Brasil altos níveis de produtividade e competitividade para atuação no mercado externo, o que justifica o bom desempenho do país em relação a exportação de alimentos industrializados (Raimundo et al., 2017). De acordo com a OECD (2013), a expansão e perspectivas de lucros das indústrias alimentícias têm atraído novos investimentos nacionais e multinacionais, atribuindo ao setor maiores vantagens competitivas. 


\subsection{Estudos Anteriores e Desenvolvimento da Hipótese de Pesquisa}

Para a perspectiva de altos retornos, os especialistas financeiros buscam mercados emergentes proeminentes, principalmente porque são mais propensos a alcançar um crescimento econômico mais rápido, medido pela taxa de crescimento do PIB, do que o das economias desenvolvidas (Rousseau \& Sylla, 2003). No entanto, os investimentos em mercados emergentes costumam acompanhar sérias incertezas devido à instabilidade política, problemas de infraestrutura, flutuação da moeda e regulamentos impostos por autoridades governamentais (Khanra \& Dhir, 2017).

Diversos estudos analisaram variáveis de risco, retorno e métricas de desempenho financeiro para avaliar a criação de valor de empresas e setores do mercado brasileiro. Rogers, Securato \& Ribeiro (2008) verificaram se empresas que adotam melhores práticas de governança corporativa possuem menor custo de capital e maior retorno do investimento e concluíram que o custo de capital e o retorno do investimento são menores para empresas com práticas de governança corporativa superiores.

No mesmo contexto, Vilhena \& Camargos (2014) concluíram que as empresas que adotaram práticas diferenciadas de Governança Corporativa são mais suscetíveis de criar valor para os acionistas do que as do mercado tradicional, mas apresentam desempenho econômico financeiro pior.

Lopes et al. (2017) analisaram a relação entre disclosure socioambiental, a reputação corporativa e a criação de valor de empresas listadas na BM\&FBovespa e rejeitaram a hipótese de que o disclosure socioambiental e a reputação corporativa são positivamente relacionados com a criação de valor, pois somente a reputação corporativa apresentou relação com a criação de valor.

Degenhart et al., (2017) analisaram a relação entre desempenho econômico e a presença de comitê de gestão de risco em empresas brasileiras. Os resultados revelaram que as empresas que possuem comitê de gestão de risco na estrutura de governança corporativa evidenciaram, no período analisado, média de desempenho econômico superior aquelas que não possuem tal comitê.

Correa et al., 2015 verificaram se a estrutura de propriedade influencia na criação de valor e no risco das empresas brasileiras de capital aberto, entre 2005 e 2011 e descobriram que a estrutura de propriedade influenciou na criação de valor e no risco, o qual acarretou efeitos na criação de valor.

Silva \& Santos (2015) estudaram a relação entre métricas tradicionais de desempenho financeiro e o impacto no valor de mercado das empresas de telefonia do Brasil presentes na BM\&FBOVESPA entre 2002 e 2012. Os resultados demonstraram que o investimento e o custo médio ponderado de capital mostraram-se significativos na explicação da variação do valor de mercado.

Corrêa et al., (2013) observaram relação direta entre EVA e direcionadores gerais de valor do mercado brasileiro (ROE, ROA, spread do acionista, margem bruta, margem líquida e giro do ativo. Além disso, na análise dos diferentes setores econômicos do mercado, por suas características específicas, encontraram-se divergências entre os indicadores financeiros considerados direcionadores de valor em cada um deles.

Aguiar et al., (2011) também investigaram se existem diferenças quanto aos direcionadores de valor em empresas brasileiras atuantes em diferentes setores e afirmaram que sim. Desse modo, sendo o setor alimentício fundamental para a economia brasileira (Raimundo et al., 2017) e diante dos estudos empíricos já apontados analisou-se a influência de indicadores de risco financeiro na criação de valor para direcionar a tomada de decisão de investidores. As hipóteses desse estudo foram definidas como:

$\mathrm{H}_{1}$ - As variáveis de risco (volatilidade e beta) impactam negativamente na criação de valor das empresas do setor alimentício. 
$\mathrm{H}_{2}$ - As variáveis de risco relacionadas com retorno (IT, IS e IR) impactam positivamente na criação de valor das empresas do setor alimentício.

\section{METODOLOGIA DE PESQUISA}

Para o desenvolvimento do trabalho realizou-se uma pesquisa de natureza descritiva e abordagem quantitativa, tendo como objetivo verificar a partir de um recorte temporal a influência do risco financeiro, entendidos nessa pesquisa como variáveis descritas por meio da mensuração de itens, na criação de valor.

\subsection{População e Amostra}

O estudo foi realizado por meio de informações econômicas e financeiras das empresas estabelecidas no Setor Alimentício e listadas na B3, entre 2013 e 2018.

Os dados foram tomados a partir do Sistema Economática ${ }^{\circledR}$, sendo que se considerou: i) resultados trimestrais contábeis; ii) DRE dos últimos 12 meses; iii) correção pela inflação (IPCA/IBGE) para agosto de 2019; iv) valores médios dos ativos financeiros (ações).

A partir da extração dos dados das empresas estabelecidas em setores da indústria alimentícia, foi possível identificar 17 empresas em agosto de 2019, contudo, para o período analisado, somente 13 empresas (Quadro 1) apresentaram volume de negócios nos seus ativos (ações) que permitiram a construção da base de dados e/ou dispunham de informações financeiras publicadas para o período, por terem ingressados na B3 durante o período de análise. Por isso, as empresas Oderich S.A., Excelsior S.A., CTC S.A. e Camil S.A. não integraram a amostra.

Quadro 1 - Amostra de empresas listadas na B3

\begin{tabular}{|l|l|}
\hline Empresa & Segmento \\
\hline Biosev & Agricultura \\
\hline Brasilagro & Agricultura \\
\hline BRF AS & Abatedouros \\
\hline J B Duarte & Indústria de alimentos \\
\hline JBS & Abatedouros \\
\hline Josapar & Moinho de grãos \\
\hline M.Diasbranco & Outras indústrias de alimentos \\
\hline Marfrig & Abatedouros \\
\hline Minerva & Abatedouros \\
\hline Minupar & Abatedouros \\
\hline Pomifrutas & Cultivo de frutas e nozes \\
\hline Sao Martinho & Indústria de açúcar e produtos de confeitaria \\
\hline SLC Agrícola & Agricultura \\
\hline
\end{tabular}

Fonte: Elaboração própria

\subsection{Variáveis e Operacionalização da Pesquisa}

Os dados foram tomados a partir do Sistema Economática ${ }^{\circledR}$, sendo que se considerou: i) resultados trimestrais contábeis; ii) DRE dos últimos 12 meses; iii) correção pela inflação (IPCA/IBGE) para agosto de 2019; iv) valores médios dos ativos financeiros (ações).

As variáveis utilizadas estão detalhadas no Quadro 2: 
Quadro 2 - Variáveis de análise

\begin{tabular}{|l|l|l|c|}
\hline Dimensão & Variáveis & Referência & Unidade \\
\hline \multirow{5}{*}{ Criação de Valor } & EVA®/Ativo & (Nekounam, Sotudeh, \& Kohandel, 2013) & $\%$ \\
\cline { 2 - 4 } & MVA®/Ativo & (Wet, 2005) & $\%$ \\
\cline { 2 - 4 } & RROI & (Corrêa, Assaf Neto e lima, 2013) & $\%$ \\
\cline { 2 - 4 } & Preço/Lucro (P/L) & (Alberto \& Vieira, 2011) & $\%$ \\
\cline { 2 - 4 } & Market to Book (M/B) & (Chen \& Zhao, 2006) & $\%$ \\
\hline \multirow{5}{*}{ Risco } & Volatilidade do ativo & (Patel, Guedes, Soares, \& Gonçalves, 2018) & $\%$ \\
\cline { 2 - 4 } & Beta & (Araújo, Oliveira, \& Silva, 2012) & $\%$ \\
\cline { 2 - 4 } & Índice de Treynor & (Treynor, 1962) & $\%$ \\
\cline { 2 - 4 } & Índice de Sharpe & (Silva, Nogueira, \& Ribeiro, 2015) & $\%$ \\
\cline { 2 - 4 } & Information Ratio & (Gaspar, Santos, \& Rodrigues, 2014) & $\%$ \\
\hline
\end{tabular}

Fonte: Elaboração própria

Optou-se pelo uso das informações reais das empresas, pois como se trata de resultados empresariais, a utilização de procedimentos de controle de outliers poderiam retirar da amostra ou minimizar o impacto de estratégias específicas das empresas, em que entende-se que são importantes que sejam conhecidas, especialmente, pelo estudo envolver as variáveis risco como explicativas.

\subsection{Instrumento de Análise dos Dados}

Por meio da utilização de indicadores financeiros para melhor compreender a gestão do valor e risco financeiro, foi utilizada a análise de regressão, a qual teve como objetivo verificar o impacto do risco nas métricas de criação de valor no setor estudado.

O modelo empírico utilizado está expresso na fórmula (1) em que foi realizado o modelo de regressão com dados em painel, conforme Greene (2003).

$$
Y_{i t}=\alpha_{i}+\beta_{1} \text { Risco }_{i t}+\beta_{2} \text { Beta }_{i t}+\beta_{3} I T_{i t}+\beta_{4} I R_{i t}+u_{i t}
$$

Os termos $i$ e $t$ denotam as empresas da amostra e o tempo, respectivamente; os $\beta(1,2,3 \mathrm{e}$ 4) são os coeficientes parciais da regressão, $\alpha$ é a constante da regressão e o termo de erro é representado por $u$. Foram realizadas 5 regressões, de modo que cada variável de criação de valor (EVA_Ativo, MVA_Ativo, P/L, P/VPA e RROI) assumiu a condição de variável dependente (Y).

Para verificar o ajuste do modelo foram utilizadas a Estatística F do modelo, o coeficiente de determinação $\mathrm{R}^{2}$ e $\mathrm{R}^{2}$ ajustado. Além desses testes, foram verificadas as premissas de homocedasticidade (Estatística Breusch-Pagan) e autocorrelação do modelo (Estatística Durbin-Watson). Em razão da presença de heteroscedasticidade, empregou-se o modelo de Mínimos Quadrados Ponderados. Foi utilizado o software Gretl para trabalhar os dados econométricos.

\section{DISCUSSÃO DOS RESULTADOS}

Após os cálculos das variáveis que compõem a análise deste estudo, foi feito um resumo quantitativo dos dados obtidos por meio de estatísticas descritivas e da matriz de correlação, a fim de compreender o comportamento destas variáveis e de que formas estão relacionadas conforme Tabela 1. 
Tabela 1 - Estatísticas Descritivas

\begin{tabular}{lccccc}
\hline & \multicolumn{3}{c}{ Estatísticas Descritivas } & & Máximo \\
Variável & Média & Mediana & Desvio Padrão & Mínimo & 2,04 \\
EVA_Ativo & $-0,0314$ & $-0,0491$ & 0,158 & $-0,447$ & $-12,1$ \\
MVA_Ativo & $-0,23$ & $-0,294$ & 1,21 & $-5,7$ & 13,62 \\
RROI & $-0,0118$ & $-0,0686$ & 0,97 & -104 & $1,25 \mathrm{E}+03$ \\
PL & 11,7 & 3,03 & 85,8 & $-43,9$ & 33,1 \\
PVPA & 1,17 & 1,05 & 5,06 & 4,57 & 140 \\
Volatilidade do Ativo & 33,1 & 27,7 & 23,3 & $-1,45$ & 4,66 \\
Beta & 0,588 & 0,418 & 0,883 & -114 & 236 \\
Índice de Treynor & 0,643 & $-0,0425$ & 17,8 & $-7,69$ & 17 \\
Índice de Sharpe & 0,0639 & $-0,63$ & 3,37 & $-7,05$ & 18,5 \\
IR & 0,561 & $-0,165$ & 3,36 & & \\
\hline
\end{tabular}

Fonte: Elaboração própria

Observa-se que as variáveis elencadas na Tabela 1 apresentam desvio padrão superior à média para todas as variáveis, exceto o risco, indicando que as empresas da amostra possuem resultados com elevada variação, o que significa que elas são diferentes entre si na gestão dos seus recursos e nas suas estratégias.

Analogamente, no que diz respeito ao comportamento das empresas, pode-se observar a variação média anual da variável Risco e da relação EVA_Ativo nas Figuras 1 e 2.

Figura 1 - Média anual da variável EVA_Ativo

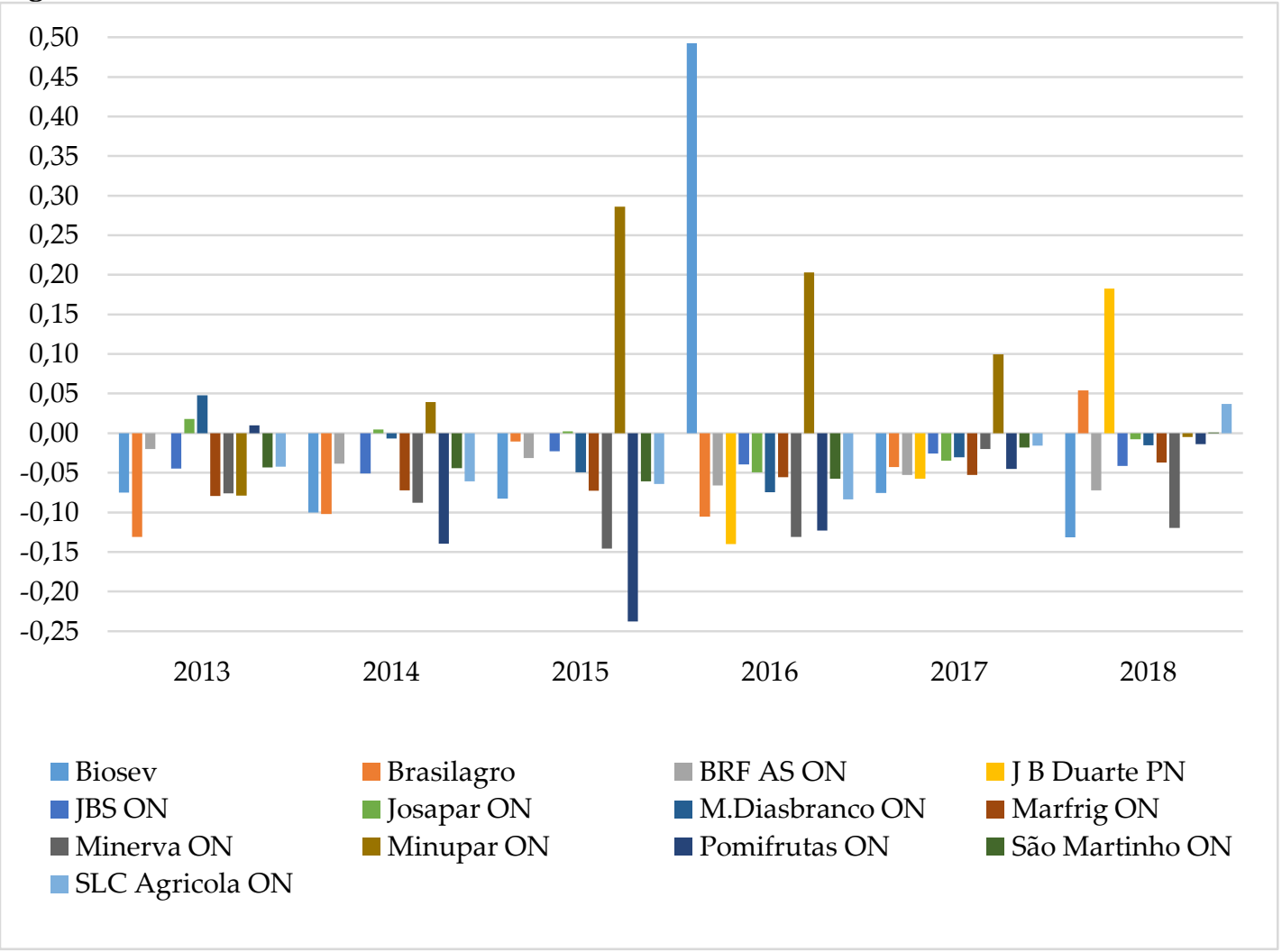

\section{Fonte: Elaboração própria}

Em relação a variável EVA_Ativo, as empresas apresentaram valores médios bem próximos durante os anos analisados, os quais se concentraram principalmente entre -0,15 e 0,05.

Para a variável Risco também se observou uma variação baixa entre os valores médios anuais, exceto 2013, conforme Figura 2. 


\section{Figura 2 - Média anual da variável Risco}

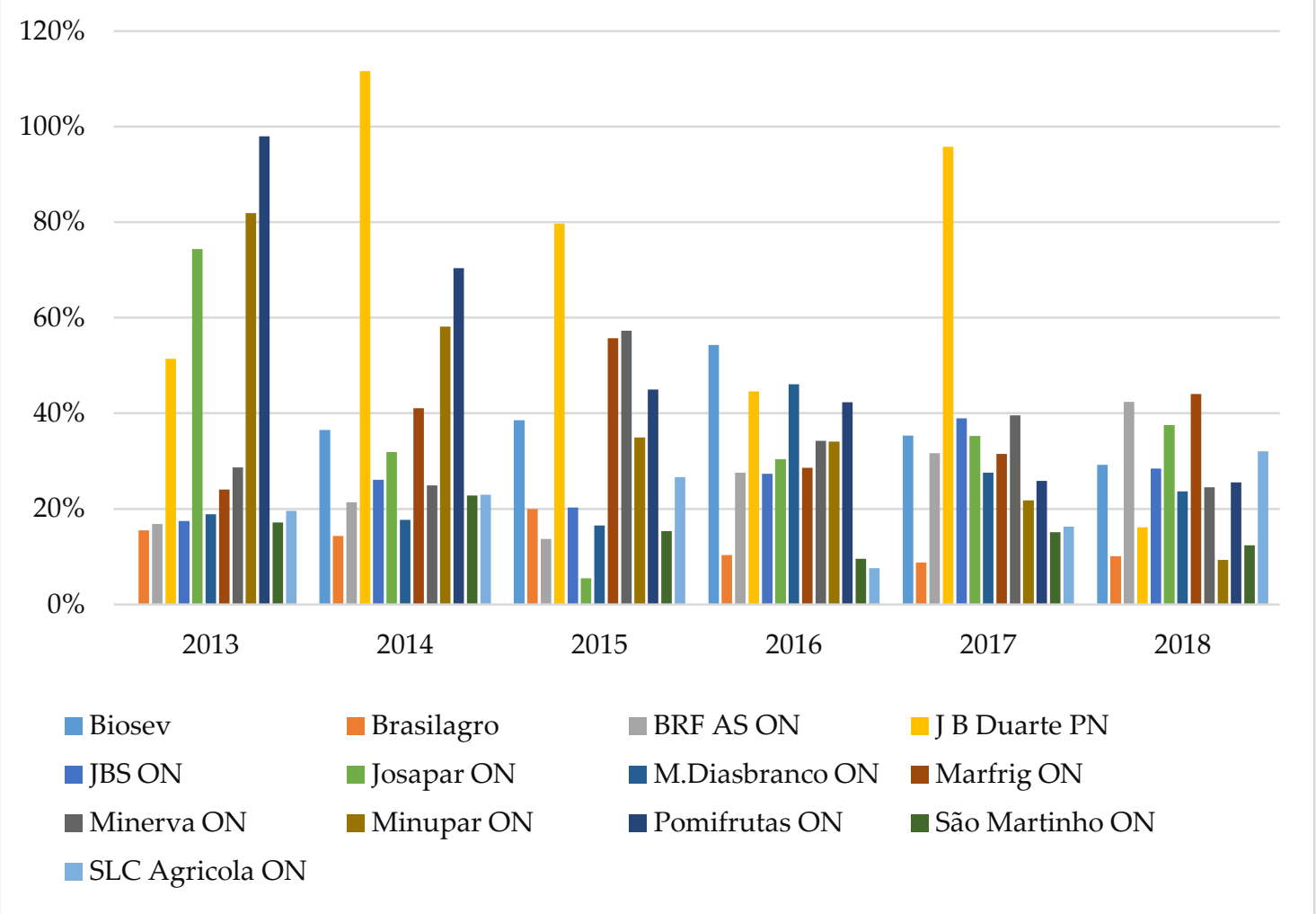

Fonte: Elaboração própria

Além disso, foi analisada a matriz de correlação (Figura 3) entre as variáveis de interesse. Verificou-se, especialmente, entre as variáveis de risco a existência de coeficientes de correlação fortes $|>0,7|$, no interesse de evitar o uso simultâneo dessas variáveis e ocasionar efeito de multicolineariedade.

Figura 3 - Matriz de correlação entre as variáveis de interesse

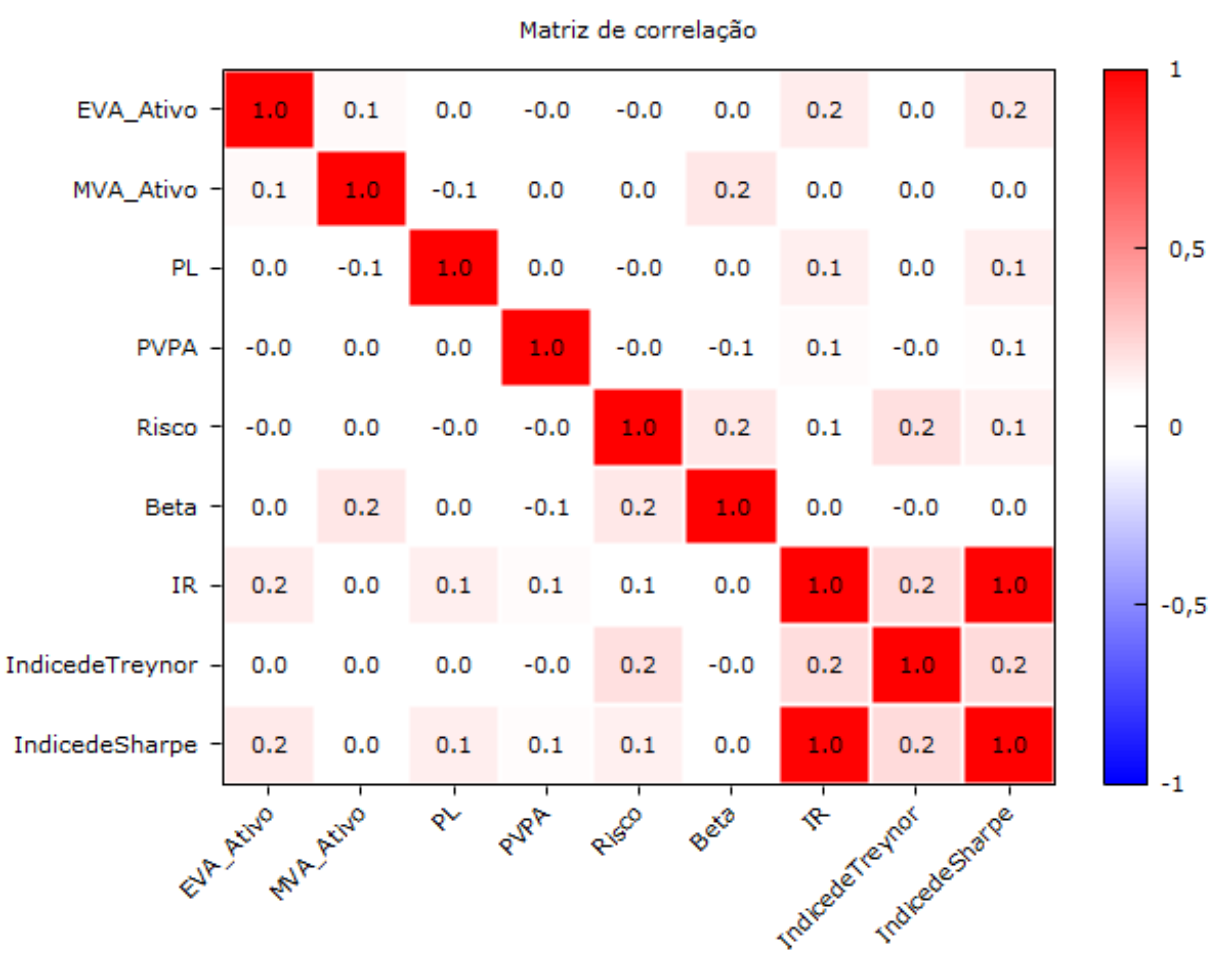

Fonte: Elaboração própria 
De acordo com a matriz de correlação, as variáveis apresentaram baixa correlação com exceção das variáveis IR e Índice de Sharpe, que resultaram em uma correlação perfeita positiva, e por este motivo, apesar de reconhecer o Índice de Sharpe como uma métrica importante para análise do risco, apenas o IR foi considerado no modelo empírico. Assim, o IS não foi utilizado, visto que apresentou elevada correlação com o IR, gerando efeito de multicolineariedade.

Para os resultados do modelo de regressão com dados em painel, foram estimados sequencialmente, o modelo de Efeitos Fixos e Efeitos Aleatórios. No entanto, há uma expectativa teórica para usar o modelo de efeitos fixos, devido as empresas serem entidades cujas trajetórias empresariais e resultados são definidas por suas escolhas, não podendo ser admitido que são decorrentes de eventos aleatórios (Carvalho et al., 2010; Brito \& Brito, 2012).

Deste modo, primeiro foram estimados os modelos de efeitos fixos para as 5 variáveis dependentes de criação de valor (Apêndice A). Os modelos não apresentaram ajuste adequado pela presença de heteroscedasticidade (Teste Wald) e autocorrelação (Durbin-Watson). É possível verificar elevada amplitude entre o $\mathrm{R}^{2}$ e o $\mathrm{R}^{2}$ ajustado revelando um possível inflacionamento do $\mathrm{R}^{2}$. A Estatística $\mathrm{F}$ também reportou a que hipótese nula de má especificação dos modelos não poderia ser rejeitada para os MVA_Ativo, P_VPA e Preço/Lucro.

A amostra limitada de informações pode ser uma possível associada a elevada diferença de resultados entre as empresas como demonstrado nos resultados descritivos pode ser a resposta à má especificação do modelo (Gujarati, 2006).

A despeito da incongruência teórica, empregou-se adicionalmente o modelo de efeitos aleatórios (Apêndice B). Para este modelo o teste Hausman rejeitou a hipótese nula de consistência dos parâmetros para a variável RROI e o teste de Breusch-Pagan rejeitou a hipótese de homocedasticidade para a variável dependente EVA_Ativo, sendo que para os demais modelos cujos testes não rejeitam a especificação do modelo não reportaram variáveis explicativas significativas.

Diante disso, optou-se pelo uso da modelagem por mínimos quadrados ponderados em painel, como estratégia para controlar os efeitos da heteroscedasticidade e autocorrel, os resultados desta modelagem encontram-se na Tabela 2.

Tabela 2 - Resultados dos modelos de regressão com dados em painel

\begin{tabular}{lccccc}
\hline \multicolumn{1}{c}{ Dependente } & EVA_Ativo & MVA_Ativo & P_VPA & Preço/Lucro & RROI \\
\hline Independentes & Coeficiente & Coeficiente & Coeficiente & Coeficiente & Coeficiente \\
Constante & $-0,0257^{*}$ & $-0,2036^{*}$ & $1,7961^{*}$ & $17,0091^{*}$ & $-0,0272^{*}$ \\
Volatilidade do Ativo & $-0,0005^{* *}$ & $-0,0026^{* *}$ & $-0,0177^{*}$ & $-0,2218^{*}$ & 0,000 \\
Beta & $-0,0160^{*}$ & $-0,0850^{* *}$ & $-0,2543^{*}$ & $-1,3660$ & $-0,0515^{*}$ \\
Índice de Treynor & 0,0001 & 0,001 & $-0,0089$ & $-0,0467$ & 0,0002 \\
IR & $0,0018^{\mathrm{b}}$ & $0,0123^{* * *}$ & $0,0525^{*}$ & $1,7139^{*}$ & 0,002 \\
$\mathrm{R}^{2}$ & 0,0819 & 0,0598 & 0,1654 & 0,2098 & 0,1561 \\
$\mathrm{R}^{2}$ Ajustado & 0,0668 & 0,0442 & 0,1514 & 0,1963 & 0,1421 \\
Estatística F & 5,4031 & 3,8483 & 11,7973 & 15,5998 & 11,1933 \\
P-Valor (F) & 0,0003 & 0,0047 & 0,0003 & 0,000 & 0,000 \\
Observações & 247 & 247 & 243 & 240 & 247 \\
\hline
\end{tabular}

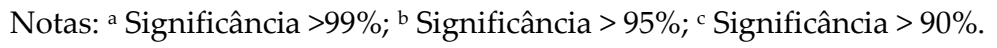

Fonte: Elaborado pela autora.

Nota-se na Tabela 2 que o modelo empírico mostrou-se adequado pela Estatística F que rejeitou a hipótese nula de má especificação e o resultado de $\mathrm{R}^{2}$ e $\mathrm{R}^{2}$ ajustados foram próximos, reduzindo a possibilidade de inflacionamento do $\mathrm{R}^{2} \mathrm{com}$ a quantidade de variáveis independentes. Pontua-se que as variáveis independentes não apresentaram multicolineariedade, o maior fator de inflação de variância foi de 1,18 para o risco, sendo este valor inferior ao máximo requerido de 10. 
Dessa forma, o modelo mostra-se ajustado para fins de avaliação do impacto das variáveis independentes, nas variáveis de criação de valor.

Ao avaliar os valores obtidos por meio deste modelo, pode-se verificar a relevância das variáveis de risco para as diferentes métricas de criação de valor, tendo em vista, que para todas as variáveis dependentes, ao menos uma variável de risco foi significativa para explicar a criação de valor ao nível igual ou superior a $90 \%$. Isso indica que as variáveis de risco são relevantes para a análise da criação de valor das empresas alimentícias que compõem a amostra de análise deste estudo.

Verifica-se na Tabela 2 que a variável Risco, enquanto volatilidade geral das ações das empresas exerce influência negativa e significativa em todas as variáveis relacionadas à criação de valor, exceto no RROI (Retorno Residual do Investimento). Assim, quanto maior a volatilidade da ação, menor a criação de valor da empresa.

Esse fato é ratificado com a segunda variável de risco utilizada, o beta $(\beta)$. Nota-se que o beta impacta negativamente todas as variáveis de criação de valor de forma significativa, exceto a variável P/L. Desse modo, tanto a volatilidade total do ativo como o risco sistemático afetam negativamente o valor das empresas.

Outro índice a ser discutido é o Information Ratio (IR) que relaciona o retorno total do ativo com a volatilidade discreta dos retornos, o qual também foi relevante para a discussão da criação de valor das empresas, pois apresentou impacto positivo e significativo em todas as variáveis de criação de valor exceto ao RROI. O impacto positivo do IR confirma que quanto maior o desempenho da relação retorno e risco da ação, maior é o potencial de criação de valor da empresa.

Com isso, visto que o IR apresentou relevância para os resultados da análise, a Hipótese 2 apresentada na metodologia - as variáveis de risco relacionadas com retorno (IT, IS e IR) impactam positivamente na criação de valor - foi parcialmente validada, uma vez que a relação positiva entre as variáveis IR e criação de valor é confirmada.

As empresas lidam diariamente com um cenário desafiador, uma vez que estão expostas a um conjunto de fatores socioeconômicos, operando de acordo com a volatilidade do mercado. Assim, as organizações desenvolvem ferramentas para avaliar os riscos e retornos dos respectivos investimentos, já que existem desafios em combinar máxima rentabilidade com baixo risco (Telo, 2001; Santos \& Coelho, 2010). Diante disso, confirma-se a Hipótese 1 desse estudo, conforme apresentado na metodologia: as variáveis de risco (volatilidade e beta) impactam negativamente na criação de valor da amostra de empresas alimentícias brasileiras analisadas nessa pesquisa.

Ademais, em qualquer negócio, quanto maior for a incerteza na sua previsão de retorno, maior será o risco ao qual este investimento estará disposto (Neves, 2000). Deste modo, uma das principais preocupações dos investidores é aferir o risco inerente às suas aplicações para determinarem (Brealey \& Myers, 1998):

- O reembolso dos valores e a possibilidade de obtenção de ganhos;

- A rentabilidade do negócio, uma vez que a remuneração deverá variar no mesmo sentido que o nível de risco associado ao investimento realizado.

Neste contexto, os resultados operacionais e financeiros das empresas, estão diretamente relacionados com a criação de valor das empresas por meio de indicadores como o EVA ${ }^{\circledR}$ e o MVA $^{\circledR}$ (Lunardi et al. 2017), os quais proporcionam um resultado que mostra se a empresa está ganhando, em um exercício, mais do que o custo de capital atribuído aos recursos utilizados. Assim, é possível avaliar quanto realmente foi agregado de valor na empresa, permitindo à gestão da empresa o discernimento exato se há criação (EVA positivo) ou destruição (EVA negativo) de valor aos acionistas (Santos et al. 2018). 
A partir disso, os resultados do modelo utilizado nesta análise indicam que as variáveis Beta, Risco e IR foram importantes para elucidar os indicadores relacionados referentes à criação de valor, comprovando a relevância do risco de mercado para as empresas.

Além disso, a criação de valor está diretamente relacionada com a capacidade da empresa em gerar retorno aos investidores que seja superior ao custo do financiamento da sua atividade (Corrêa et al., 2013; Sila \& Santos 2015). Logo, tal como referido anteriormente, quanto maior for o risco percepcionado pelos investidores relativamente à empresa, maior o custo que vão exigir para remunerar os capitais que aplicam na sua atividade.

Em um ambiente competitivo os gestores buscam estratégicas que maximizem o retorno para o acionista, sendo que para um nível maior de risco espera-se obter retorno adicional que ultrapasse o custo do investimento gerando valor agregado ao negócio (Aguiar et al., 2011). Nesse aspecto, o impacto das variáveis de risco e desempenho nas métricas de criação de valor de mercado como o P/L e P/VPA, apontam para a perspectiva de que as empresas com maior potencial de criação de valor são aquelas que proporcionam menores taxas de riscos aos seus acionistas, fato que também é confirmado a partir da análise do IR.

A partir disso, evidencia-se que as empresas com maiores índices de riscos não são capazes de proporcionar melhores remunerações aos seus acionistas, ao contrário, as empresas que apresentam menores níveis de volatilidade são aquelas que em geral oferecem melhores resultados para os acionistas. Sendo assim, o investimento de capital está relacionado ao processo que consiste em analisar as alternativas de dispêndio de capital diante da variedade de opções disponíveis no mercado considerando as probabilidades de um ativo gerar valor e proporcionar retorno aos acionistas acima ou abaixo da média esperada, resultando em lucro ou prejuízo financeiro (Silva et al., 2012).

Com isso, as empresas que possuem capacidade de criação de valor superior à de seus concorrentes apresentam vantagem competitiva no mercado, ao passo que a superioridade na criação de valor propicia capacidade de manobra e estratégias de competitividade (Brito \& Brito, 2012).

Diante dos resultados da pesquisa, torna-se possível apontar as implicações teóricas e gerenciais que contribuem no melhor entendimento da relação entre o risco e o valor nas indústrias alimentícias, relação ainda não explorada plenamente em trabalhos empíricos.

\section{CONCLUSÃO}

Duas hipóteses foram analisadas neste estudo. A hipótese 1 propõe que as variáveis de risco (volatilidade e beta) impactam negativamente na criação de valor. Já a hipótese 2, parte do pressuposto de que as variáveis de risco relacionadas com retorno (IT, IS e IR) impactam positivamente na criação de valor.

Os resultados da pesquisa confirmam a Hipótese 1. Nesse escopo, verificou-se a relevância das variáveis de risco para as diferentes métricas de criação de valor, tendo em vista, que para todas as variáveis dependentes, ao menos uma variável de risco foi significativa para explicar a criação de valor ao nível igual ou superior a 90\%. Isso indica que as variáveis de risco são relevantes para a análise da criação de valor das empresas alimentícias que compõem a amostra de análise deste estudo.

A Hipótese 2 foi parcialmente validada, uma vez que a relação positiva entre as variáveis IR e criação de valor é confirmada, enquanto as demais não foram significantes de acordo com o modelo apresentado.

Nesse aspecto, a variável Risco, enquanto volatilidade geral das ações das empresas, exerce influência negativa e significativa em todas as variáveis relacionadas à criação de valor, exceto no RROI. Dessa forma, conclui-se que quanto maior a volatilidade da ação, menor a criação de valor da empresa. 
As implicações gerenciais do estudo revelam que - em especial na Hipótese 1 - maior atenção deve ser apontada para mitigar o risco e consequentemente maximizar a criação de valor. Para tal, a implementação de melhorias dos aspectos relacionados à governança, ética e transparência do negócio como estratégia podem reduzir as assimetrias e os impactos do risco da indústria alimentícia.

Quanto às contribuições teóricas, o estudo mostra que a criação de valor deve ser o objeto principal da gestão financeira de uma empresa e é a partir do conhecimento do valor de um negócio que é possível tomar diversas decisões, as quais incluem: atrair investidores para as ações da empresa, atrair assessoria de banco de investimento para captação de recursos, escolher se é melhor aumentar o investimento na empresa ou investir em uma nova, entre outras.

O desenvolvimento deste estudo apresentou algumas limitações. No que se refere ao tempo, os dados apresentados referem-se a um período específico de tempo tomado para a análise das empresas apresentadas. Já em relação ao contexto, os resultados são restritos às empresas alimentícias que foram foco deste estudo, de modo que aplicações para outros setores precisam ser feitas com ressalvas. E por fim, quanto às características das empresas, não se deve replicar os resultados e conclusões deste estudo para empresas de capital fechado, ou até mesmo de capital aberto deste setor ou de outros que não contemplam a amostra.

Como sugestão para trabalhos futuros, propõem-se: i) Explorar demais fatores que podem influenciar o risco inerente às empresas, o qual pode ser mensurado de diferentes formas proporcionando variabilidade de resultados relacionados aos aspectos endógenos e exógenos às empresas; ii) Explorar as dimensões de criação de valor e retorno, analisando de que forma se relacionam nos resultados e desempenho das empresas, buscando identificar as principais implicações e diferenças entre ambas; iii) Avaliar o comportamento do risco nos diferentes segmentos da indústria alimentícia.

\section{REFERÊNCIAS}

Alberto, J. G. C., \& Vieira, B. L. S. (2011). Análise do retorno de ações com baixo Preço/lucro e boa liquidez na Bovespa no período 1999 a 2007. Revista Contabilidade e Controladoria, 3(3).

Aguiar, A. B., Pimentel, R. C., Rezende, A. J., \& Corrar, L. J. (2011). Análise dos direcionadores de valor em empresas brasileiras. Revista de Administração Mackenzi, 12, 90-112.

Araújo, E. A., Oliveira, V. D., \& Silva, W. A. (2012). CAPM em Estudos Brasileiros: Uma Análise da Pesquisa. Revista de Contabilidade e Organizações, 6(15), 95-122.

Araújo, A. M. P. D., \& Assaf Neto, A. (2003). A contabilidade tradicional e a contabilidade baseada em valor. Revista Contabilidade \& Finanças, 14(33), 16-32.

Bali, T. G., \& Cakici, N. (2009). World market risk, country-specific risk and expected returns in international stock markets. Journal of Banking \& Finance, 34, 1152-1165.

Bali, T. G., \& Zhou, H. (2016). Risk, Uncertainty, and Expected Returns. Journal of Financial and Quantitative Analysis, 51(3), 707-735.

Bartram, S. M., Brown, G. W., \& Waller, W. (08 de 2015). How Important Is Financial Risk? Journal of Financial and Quantitative Analysis, 50(4), pp. 801-824.

Biasi, N. B., Wiese, A. L. H. C., Caron, A., \& Seleme, R. (2018). Inovação como vantagem competitiva na indústria alimentícia. Revista da FAE, 21(2), 7-20.

Brealey, R., \& Myers, S. (1998). Princípios de finanças empresariais. Porto Alegre: AMGH. 
Bhimani, A. (2009). Risk management, corporate governance and management accounting: Emerging interdependencies. 2(5).

Brito, R. P., \& Brito, L. A. (2012). Vantagem competitiva, criação de valor e seus efeitos sobre o desempenho. Revista de Administração de Empresas, 52, 70-84.

Carvalho, F. M., Kayo, E. K., \& Martin, D. M. (2010). Tangibilidade e intangibilidade na determinação do desempenho persistente de firmas brasileiras. Revista de Administração Contemporânea, 14, 871-889.

Chen, L., \& Zhao, X. (2006). On the relation between the market-to-book ratio, growth opportunity, and leverage ratio. Finance Research Letters, 3(4), pp. 253-266.

Corrêa, A. C. C., Assaf Neto, A., \& Lima, F. G. (2013). Os indicadores financeiros tradicionais explicam a geração de valor no Brasil? Um estudo empírico com empresas não financeiras de capital aberto. Práticas em Contabilidade e Gestão, 1(1).

Costa, C. C., Almeida, A. L., Ferreira, M. A., \& Silva, E. A. (2013). Determinantes do Desenvolvimento do setor Agropecuáro nos Municípios. Revista de Administração, 295-309.

Degenhart, L., Leite, M., Silva, A. D., Silva, M. Z. D., \& Fernandes, F. C. (2017). Relação entre presença de comitê de gestão de risco e desempenho econômico em empresas brasileiras. Revista de Gestão dos Países de Língua Portuguesa, 16(1), 4-21.

Fama, E. F., \& French, K. R. (2004). The Capital Asset Pricing Model: Theory and Evidence. Journal of Economic Perspectives, 18(3), pp. 25-46.

Farina, E. M. (1999). Competitividade e Coordenação de Sistemas Agroindustriais: um ensaio conceitual . Gestão e Produção , 6, 147-161.

Gaspar, B. C., Santos, D. F., \& Rodrigues, S. V. (2014). Risco Versus Retorno das Ações do Setor Imobiliário da BM\&FBOVESPA no Período de 2009 a 2012. Revista Eletrônica de Ciência Administrativa, 13, 326-338.

Gouveia, F. (2006). Indústria de alimentos: no caminho da inovação e de novos produtos. Inovação Uniemp, 2(5), 32-37.

Gujarati, D. (2006). Econometria Básica. Porto Alegre: Bookman.

Kapustina, N., Rjachovskaya, A., Rjachovskij, D., \& Gantseva, L. (2018). External risk factors influence on the financial stability of construction companies. Journal of Reviews on Global Economics, 7(Special Issue), 726-730.

Kayo, E. K. (2015). Réplica 1-Crescimento Organizacional, Tamanho das Firmas e Valor Econômico. Revista de Administração Contemporânea, 19(1), 142-148.

Khanra, S., \& Dhir, S. (2017). Creating value in small-cap firms by mitigating risks of market volatility. Vision, 21(4), 350-355.

Krücken-Pereira, L., de Abreu, A. F., \& Bolzan, A. (2002). A necessidade de inovar: um estudo na indústria de alimentos. Revista de Ciências da Administração, 4(6), 19-27.

Lopes, A. C., De Luca, M. M. M., Góis, A. D., \& de Vasconcelos, A. C. (2017). Disclosure socioambiental, reputação corporativa e criação de valor nas empresas listadas na bm\&fbovespa. Revista Ambiente Contábil, 9(1), 364-382. 
Lunardi, M. A., Barbosa, E. T., Junior, M. M. R., da Silva, T. P., \& Nakamura, W. T. (2017). Criação de valor no desempenho econômico de empresas familiares e não familiares brasileiras. Revista Evidenciação Contábil \& Finanças, 5(1), 94-112.

Luz, E. R. (2013). Indicadores tradicionais de liquidez e de rentabilidade: Um estudo comparativo da média setorial das empresas brasileiras nos anos 2000 a 2010. Revista ADMPG, 6(2).

Martins, E. (2009). Avaliação de Empresas: Da mensuração Contábil à economia (1 ed.). São Paulo: Atlas.

Nekounam, J., Sotudeh, R., \& Kohandel, Z. (2013). Explain the relationship between corporate governance on economic value-added (EVA) and created shareholder value (CSV). Life Science Journal, 10(2), 67-73.

Neves, J. C. (2000). Análise Financeira Volume I - Técnicas Fundamentais.

OECD. (2013). Competition issues in the food chain industry. Fonte: http://www.oecd.org/daf/competition/CompetitionIssuesintheFoodChainIndustry.pdf

Oliveira Filho, B. G., \& Sousa, A. F. (2015). Fundos de Investimento em Ações no Brasil: Métricas para Avaliação de Desempenho. Revista de Gestão, 22(1), 61-76.

Patel, P., Guedes, M., Soares, N., \& Gonçalves, V. (2018). Strength of the association between R\&D volatility and firm growth: The roles of corporate governance and tangible asset volatility. Journal of Business Research, 88, 282-288.

Raimundo, L. M., Batalha, M. O., \& Torkomian, A. L. (2017). Dinâmica tecnológica da Indústria Brasileira de Alimentos e Bebidas (2000-2011). Gest. Prod., 24(2). 423-436.

Rogers, P., Securato, J. R., \& de Sousa Ribeiro, K. C. (2008). Governança corporativa, custo de capital e retorno do investimento no Brasil. REGE Revista de Gestão, 15(1), 61-77.

Rousseau, P. L., \& Sylla, R. (2003). Financial systems, economic growth, and globalization. In Globalization in historical perspective. University of Chicago Press. 373-416.

Santos, J. V. J., Tavares, A. L, Azevedo, Y. G. P., \& de Freitas Neto, R. M. (2018). Relação entre o retorno das ações e o economic value added (EVA): evidências empíricas em companhias abertas no Brasil. Revista Evidenciação Contábil \& Finanças, 6(1), 119-131.

Santos, J. O.; Watanabe, R. (2005) Uma análise da Correlação entre o EVA® e o MVA® no contexto das empresas brasileiras de capital aberto. REGE Revista de Gestão, 12(1). 19-32.

Santos, J. O., \& Coelho, P. A. (2010). Análise da relação risco e retorno em carteiras compostas por índices de bolsa de valores de países desenvolvidos e de países emergentes integrantes do bloco econômico BRIC. Revista Contabilidade e Finanças, 21, 23-37.

Scagliusi, F., Machado, F., \& Torres, E. (2005). Marketing aplicado à indústria de alimentos. Nutrire: Rev. Soc. Bras. Alim. Nutr., 30, 79-95.

Silva, B. A., Nogueira, S. G., \& Ribeiro, K. C. (2015). Aplicação Prática do Índice de Sharpe na Determinação de um Portfólio Ótimo de Ativos. Revista Eletrônica de Administração, 14(26), 8599.

Silva, D. L., Motta, P. D., Ferreira, M. S. Â., Duarte, M. B. A., \& de Oliveira, M. F. (2018). Gestão baseada no valor. LIBERTAS: Revista de Ciênciais Sociais Aplicadas, 8(2), 207-225. 
Silva, R. L., Nardi, P. C., \& Pimenta Junior, T. (2012). O Impacto da Migração das Empresas para os Níveis Diferenciados de Governança Corporativa da BM\&FBOVESPA sobre o Risco e o Retorno de suas Ações. Revista de Administração da UFSM, 5(2), 222-242.

Silva, C. T. R., \& Santos, D. F. L. (2015). Desempenho financeiro e valor de mercado do setor de telefonia no Brasil. Revista Ciências Administrativas, 21(1), 42-67.

Silveira, A. G., Santos, D. F., \& Rodrigues, S. V. (2017). Análise do desempenho das ações do setor do agronegócio na BM\&FBOVESPA. Revista de administração IMED, 7(1).

Teixeira, N. M., \& Amaro, A. G. (2013). Avaliação do desempenho financeiro e da criação de valor - um estudo de caso. Revista Universo Contábil, 9(4), 157-178.

Telo, A. (2001). Desempenho Organizacional: Planejamento Financeiro em Empresas Familiares. Revista FAE, 4, 17-26.

Tobin, J. (1969). A general equilibrium approach to monetary theory. Journal of money, credit and banking, 1(1), 15-29.

Toledo, A. G., \& Mello, R. B. (2013). Criação de Valor: Incorporando Elementos da Economia dos Custos de Transação na Visão Porteriana. Revista de Administração Contemporânea, 17, 285303.

Treynor, J. L. (1962). Toward a Theory of Market Value of Risky Assets, Unpublished manuscript. A final version was published in 1999, in. Asset Pricing and Portfolio Performance: Models, Strategy and Performance Metrics.Robert A. Korajczyk (editor) London: Risk Books, p. 1522.

Varga, G. (2001). Índice de Sharpe e Outros Indicadores de Performance Aplicados a Fundos de Ações Brasileiros. Revista de Administração Contemporânea. Rio de Janeiro, 5(3), 215-245.

Vilhena, F. A. C., \& Camargos, M. A. (2015). Governança corporativa, criação de valor e desempenho econômico-financeiro: evidências do mercado brasileiro com dados em painel, 2005-2011. REGE-Revista de Gestão, 22(1), 77-96.

Wet, J. (2005). EVA versus traditional accounting measures of performance as drivers of shareholder value - A comparative analysis. Meditari Accountancy Research, 13(2), 1-16. 


\section{Apêndice A}

Resultados modelo empírico, considerando a técnica de Efeito Fixo

\begin{tabular}{lccccc}
\hline \multicolumn{1}{c}{ Variáveis } & \multicolumn{5}{c}{ Dependentes } \\
\hline \multicolumn{1}{c}{ Independentes } & EVA_Ativo & MVA_Ativo & P_VPA & Preço/Lucro & RROI \\
\hline Constante & $-0,00339131$ & $-0,444543^{\mathrm{b}}$ & $1,46109^{\mathrm{a}}$ & $16,8325^{\mathrm{a}}$ & $-5,53016^{\mathrm{a}}$ \\
Volatilidade do Ativo & $-0,00071643$ & 0,00444081 & $-0,00551198$ & $-0,196626$ & 0,0787147 \\
Beta & $-0,0219051$ & 0,133018 & $-0,180226$ & $-1,06668$ & $-0,0515^{\mathrm{a}}$ \\
Índice de Treynor & $-6,80045 \mathrm{e}-05$ & 0,00207927 & 0,000470 & $-0,100943$ & $-0,0186776$ \\
IR & $0,0103078^{\mathrm{a}}$ & 0,00831786 & 0,142839 & 3,75816 & $-0,0681900$ \\
$\mathrm{R}^{2}$ & 0,178670 & 0,0966 & 0,068130 & 0,075966 & 0,279889 \\
$\mathrm{R}^{2}$ Ajustado & 0,049238 & 0,0126 & 0,008239 & 0,018017 & 0,085223 \\
Estatística F & 3,127104 & 1,537983 & 1,032691 & 1,145812 & 5,514327 \\
P-Valor (F) & 0,000078 & 0,087787 & 0,423048 & 0,314375 & $6,36 \mathrm{e}-10$ \\
Teste Wald $\left(X^{2}\right)$ & $2938,1(0,00)$ & $51655,1(0,00)$ & $6,91 \mathrm{e}+006(0,00)$ & $1,63 \mathrm{e}+006(0,00)$ & $904194(0,00)$ \\
Durbin-Watson & 1,719376 & 1,661475 & 2,582182 & 1,214952 & 0,942633 \\
Observações & 247 & 247 & 243 & 240 & 247 \\
\hline
\end{tabular}

\section{Apêndice B}

Resultados modelo empírico, considerando a técnica de Efeito Aleatório

\begin{tabular}{lccccc}
\hline \multicolumn{1}{c}{ Variáveis } & \multicolumn{5}{c}{ Dependentes } \\
\hline \multicolumn{1}{c}{ Independentes } & EVA_Ativo & MVA_Ativo & P_VPA & Preço/Lucro & RROI \\
\hline Constante & $-0,00846015$ & $-0,375409^{\mathrm{b}}$ & $1,66487^{\mathrm{a}}$ & 16,5861 & 0,108956 \\
Volatilidade do Ativo & $-0,00055192$ & $9,36775 \mathrm{e}-05$ & $-0,0102333$ & $-0,223994$ & $-0,00117025$ \\
Beta & $-0,0136490$ & $0,248725^{\mathrm{b}}$ & $-0,309913$ & 0,826830 & $-0,153292^{\mathrm{c}}$ \\
Índice de Treynor & $-1,37593 \mathrm{e}-05$ & 0,00104835 & $-0,00885818$ & $-0,0910170$ & $0,00712843^{\mathrm{b}}$ \\
IR & 0,00930257 & 0,0117646 & 0,166248 & $3,85018^{\mathrm{b}}$ & $-0,000651132$ \\
Teste Breusch-Pagan & $19,0621(0,0)$ & $0,420655(0,52)$ & $0,0084676(0,92)$ & $0,0947079(0,76)$ & $0,959168(0,33)$ \\
Teste Hausman & $3,34546(0,5)$ & $5,73409(0,22)$ & $4,21526(0,38)$ & $0,504493(0,97)$ & $17,0532(0,002)$ \\
Observações & 247 & 247 & 243 & 240 & 247 \\
\hline
\end{tabular}

